\title{
Protocol
}

\section{Psychometric properties of quantitative sensory testing focusing on healthy and patients with shoulder pain: a systematic review protocol}

\author{
Paraskevi Bilika $^{1 *}$, Konstantina Savvoulidou', Achilleas Paliouras ${ }^{1}$, Zacharias Dimitriadis ${ }^{2}$, \\ Evdokia Billis $^{3}$, Nikolaos Strimpakos ${ }^{2,4}$, Eleni Kapreli ${ }^{1}$
}

\begin{abstract}
${ }^{1}$ Department of Physiotherapy, Clinical Exercise Physiology and Rehabilitation Laboratory, University of Thessaly, Greece

${ }^{2}$ Department of Physiotherapy, Health Assessment and Quality of Life Laboratory, University of Thessaly, Greece ${ }^{3}$ Department of Physical Therapy, School of Health Rehabilitation Sciences, University of Patras, Aigio, Greece

${ }^{4}$ Division of Musculoskeletal \& Dermatological Sciences, The University of Manchester, Manchester, UK
\end{abstract}

Received: 05 July 2021

Revised: 19 July 2021

Accepted: 20 July 2021

\section{*Correspondence: \\ Paraskevi Bilika, \\ E-mail: pbilika@uth.gr}

Copyright: (C) the author(s), publisher and licensee Medip Academy. This is an open-access article distributed under the terms of the Creative Commons Attribution Non-Commercial License, which permits unrestricted non-commercial use, distribution, and reproduction in any medium, provided the original work is properly cited.

\section{ABSTRACT}

Background: Quantitative sensory testing (QST) is a battery of non-invasive psychophysical methods to assess the function of somatosensory system. Although the use of QST is widespread and several studies in patients with chronic shoulder pain have used it, the level of evidence for the psychometric properties has not been established. The aim of this protocol is to investigate, through a systematic review, the level of evidence for the psychometric properties of QST in the shoulder.

Methods: For conducting and reporting this review the preferred reporting items for systematic reviews and metaanalysis (PRISMA) guidelines and the consensus-based standards for the selection of health measurement instruments (COSMIN) guidelines will be used. Nine databases including PubMed, Medline, AMED (via EBSCO), PEDRO, Embase, Web of Science, Scopus, SportDiscus, Google Scholar and Cochrane Library will be searched for the period from their inception until September 2021. Two reviewers (BP and SK) will independently evaluate the retrieved articles (titles and abstracts) and the psychometric characteristics checklist based on the standards from the COSMIN. The modified grading of recommendations assessment, development, and evaluation (GRADE) approach will be used to assess the overall quality of the evidence.

Conclusions: Evaluation of the level of evidence for the psychometric properties of QST in the shoulder is an essential step for evidence-based assessment in clinical practice.

Trial registration: PROSPERO registration number is CRD42021232778.

Keywords: Quantitative sensory testing, Shoulder, Psychometric properties

\section{INTRODUCTION}

Shoulder pain (SP) is one of the most common musculoskeletal disorders and increases with age. ${ }^{1}$ Shoulder includes a variety of structures, and it has a wide range of motion, thus its dysfunction causes serious consequences for patients, and it is a challenge for health professionals. $^{2}$ A recent study shows that the severity of shoulder pain is a risk factor for poor physical quality of like in middle-aged and elderly people. ${ }^{3}$ The high levels of recurrence and chronicity of shoulder pain drove scientists away from the structural model of pain. It is now accepted that the extent of structural damage is often unrelated to the experience of pain. ${ }^{4,5}$ Over the past decade, a neurophysiological mechanism-based approach has been proposed for the diagnosis and management of chronic 
pain. ${ }^{6}$ The clinical features of patients can provide important information about the underlying pathophysiological mechanisms., ${ }^{7,8}$ However, clinical evaluation seems to lag in detecting the mechanisms that contribute to the perception of pain. ${ }^{9}$ Thus, it is recommended to use objective measurements that will help strengthen the diagnosis and the direction of treatment.

Quantitative sensory testing (QST) is a battery of noninvasive psychophysical methods to assess the function of somatosensory system. ${ }^{10}$ QST evaluates subjective responses to a controlled quantitative stimulus. The procedure may involve evaluating the minimum perceived pain threshold, locating the stimulus, the threshold at which the stimulus becomes painful, the tolerance or differentiation of different sensory stimuli and is carried out with the use of simple tools for assessing perception of touch, vibration, proprioception, pinprick/blunt pressure sensitivity or sensitivity to cold or heat stimuli. ${ }^{11}$ Table 1 presents different parameters of QST, the type of stimuli applied and the type of fibers stimulated. ${ }^{10,12,13}$ The diversity of QST provides the advantage of estimating sensory processing by large $(\mathrm{A} \alpha$ and $\mathrm{A} \beta)$ and small $(\mathrm{A} \delta$ and $C$ ) afferent nerve fibers and can provide important information about pain mechanisms. ${ }^{12}$ It has been observed that a subset of patients with shoulder pain develop central sensitization signs such as generalized hyperalgesia, diffuse pain, and other centrally maintained symptoms. ${ }^{14-16}$ QST may provide: indications of peripheral or central sensitization (different response in affected or non-affected area); information on changing pain sensitivity in patients (compared to healthy individuals) but also between patients, possibly reflecting the mechanisms of pain, (i.e. sensitivity following the course of a nerve or diffuse, extensive pain without neuroanatomical logical); prediction of treatment outcomes; and results on response to treatment. ${ }^{12,17-21}$

In an attempt to detect prognostic factors for the recognition of central sensitization, the systematic review of Clark et al showed that there is moderate evidence of a higher risk of developing central sensitization when there were higher levels of sensory sensitivity in the patient's history. ${ }^{22}$ A recent systematic review revealed a link between pain sensitivity, using QST, and disability. Also, QST may predict other factors such as depression in patients with musculoskeletal pain. ${ }^{23}$ Although the use of QST is widespread and several studies in patients with chronic shoulder pain have used it, there is inconsistency in the results due to different protocols and other factors that may affect measurements (stimulation parameters, detection of stimuli, order of testing procedures, raters training, participants' familiarization, medication usage etc.). ${ }^{24-26}$ However, the use of reliable and valid assessment tools is vital whether they are used for research or clinical purposes. Over the last years, systematic reviews have been published that have examined the psychometric properties (PMPs) of different components of QST in different populations. It seems that there is great variability in the reliability of specific sensory tests (thermal stimuli) as opposed to other tests (such as conditioning pain modulation-CPM). ${ }^{25,27}$ There is also, great heterogeneity in the protocols and population of studies. ${ }^{28}$ It is worth mentioning that, in order to use a sensory test, its PMPs should be evaluated at a specific site for the target population. QST is used in adjunct with clinical decision making and therefore must be accurate when applied. A systematic review evaluated the level of studies focusing on the PMPs of the sensory abnormalities' assessment in individuals with pain in the joints of the upper and lower extremities until 2016. There is a lack of research on PMPs of QST test procedures in shoulder, elbow, hip, and ankle joint pain. The authors concluded that improvements in the quality of primary studies are needed as risks of bias are identified that threaten the validity of the results. ${ }^{24}$

Table 1: Parameters of QST.

\begin{tabular}{|lll|}
\hline $\begin{array}{l}\text { QST parameter } \\
\begin{array}{l}\text { Pressure pain } \\
\text { threshold }\end{array}\end{array}$ & $\begin{array}{l}\text { Type of } \\
\text { stimulus }\end{array}$ & $\begin{array}{l}\text { Nerve } \\
\text { fiber }\end{array}$ \\
\hline $\begin{array}{l}\text { Mechanical } \\
\text { detection threshold }\end{array}$ & Mechanical & $\mathrm{A} \delta$ and C \\
\hline $\begin{array}{l}\text { Temporal pain } \\
\text { summation }\end{array}$ & Mechanical & $\mathrm{A} \delta$ and C \\
\hline $\begin{array}{l}\text { Sharp pain } \\
\text { threshold }\end{array}$ & Mechanical & $\mathrm{A} \delta$ \\
\hline $\begin{array}{l}\text { Conditioned pain } \\
\text { modulation }\end{array}$ & $\begin{array}{l}\text { 2 stimuli } \\
\text { (mechanical+isc }\end{array}$ & $\mathrm{A} \beta$ and C \\
\hline $\begin{array}{l}\text { hibration threshold } \\
\text { Light touch } \\
\text { threshold }\end{array}$ & Mechanical & $\mathrm{A} \beta$ \\
\hline $\begin{array}{l}\text { Warm pain } \\
\text { threshold }\end{array}$ & Mechanical & $\mathrm{A} \beta$ \\
\hline $\begin{array}{l}\text { Warm detection } \\
\text { threshold }\end{array}$ & Thermal & $\mathrm{A} \delta$ and C \\
\hline $\begin{array}{l}\text { Cold detection } \\
\text { threshold }\end{array}$ & Thermal & $\mathrm{C}$ \\
\hline Heat pain threshold & Thermal & $\mathrm{A} \delta$ \\
\hline Cold pain threshold & Thermal & $\mathrm{A} \delta$ and $\mathrm{C}$ \\
\hline $\begin{array}{l}\text { Electrical } \\
\text { perception/pain } \\
\text { threshold }\end{array}$ & Electrical \\
\hline $\begin{array}{l}\text { Flexor withdrawal } \\
\text { reflex threshold }\end{array}$ & Electrical & $\mathrm{A} \delta$ \\
\hline
\end{tabular}

At present, there are no recent systematic reviews (or meta-analyzes) investigating the PMPs of QST specifically targeting the shoulder. Therefore, it is advisable to create a systematic review and meta-analysis to investigate the PMPs of QST in shoulder. The current protocol presents the methods and process for estimating the levels of evidence of the QSTs psychometric studies in shoulder. 


\section{METHODS}

This systematic review and metanalysis will follow the preferred reporting items for systematic reviews and metaanalysis (PRISMA) guidelines and the consensus-based standards for the selection of health measurement instruments (COSMIN) guidelines (https://www.cosmin. $\mathrm{nl} /$ tools/checklists-assessing-methodological-studyqualities/) for conducting and reporting the review, international prospective register of ongoing systematic reviews (PROSPERO) registration, http://www.crd. york.ac.uk/prospero. ${ }^{29,30}$

\section{Eligibility criteria}

This systematic review will focus on studies evaluating the psychometric parameters of QST in the shoulder. Therefore, due to the nature of the studies that will be included in this work, the eligibility criteria are determined by the parameters: population, construct, outcome measures, study type. Any studies excluded, will be reported on the PRISMA flow diagram.

\section{Inclusion criteria}

\section{Population}

Adults ( $\geq 18$ years old), asymptomatic participants or with musculoskeletal shoulder pain. There will be no restrictions in terms of gender and/or ethnicity.

\section{Construct}

Studies involving one or more QST components (chemical, electrical, mechanical, and/or thermal stimuli applied to the skin, muscles, or joints) in the shoulder area.

\section{Outcome measures}

Quantitative studies considering any of the following psychometric properties (PMPs) of QST (total protocol or part of this) in the shoulder-reliability (absolute reliability (agreement) and relative reliability of testretest, intra-rater and/or inter-rater designs), validity (criterion (concurrent or predictive), construct (hypothesis testing - known-groups or comparison with other outcome measurements), responsiveness, specificity and sensitivity.

\section{Study design}

Studies including PMPs of QST (total protocol or part of this) in the shoulder as one of the (primary) aims or the sole aim of their study; studies that have valued reliability, validity, specificity, sensitivity and/or responsiveness as secondary or addible findings, (in this case, the full text will be considered and accepted only if quality/bias risk assessment information is available); peer-reviewed observational studies, cross-sectional studies, randomized controlled trials, controlled clinical trials will be included if they have at least one PMP of QST (at least one component of QST); studies in English and Greek will be accepted to ensure time and cost. The full text of the studies to be included should also be available.

\section{Exclusion criteria}

Studies with experimental pain or symptoms; duplicate publication of data (follow-up analysis of already published data); studies including participants with neurological disorders including cerebral, spinal cord, basal ganglia, brainstem, cerebellar and peripheral nerve injuries and/or diseases; and data and results from abstracts of conference presentations, systematic reviews and metaanalyses, narrative reviews, book reviews, book chapters, doctoral dissertations, other dissertations theses, case series/reports, commentaries, editorials, letters to the editor, patient education handouts, consensus statements, clinical practice guidelines, or unpublished literature were excluded from the study.

\section{Information sources}

Searches will include the following databases: PubMed, Medline, AMED (via EBSCO), PEDRO, Embase, Web of Science, Scopus, SportDiscus, Google Scholar and Cochrane Library for the period from their inception until September 2021. Up-to-date and comprehensive search strategies will be developed, based on database suggestions to ensure search effectiveness.

\section{Search strategy}

To ensure the maximum possible search results, the initial search of the databases will be performed without the restrictions (filters) on language or date of publication. A professor in the field of physiotherapy from the University of Thessaly and experience with previous systematic reviews will contribute to the search. The search strategy will include topic headings and keywords, combining $\mathrm{MeSH}$ terms related to psychometric attributes (reliability, validity, responsiveness, specificity, sensitivity), quantitative sensory testing (PPT, CMP, etc.) and shoulder. We also checked the reference lists from relevant articles to contribute to the completeness of the search. ${ }^{23,24}$ The article selection procedure will be presented in the PRISMA flowchart. A complete electronic search strategy for the PubMed database is presented in Table 2. This search strategy will be adapted to the rest of the databases.

\section{Study selection}

Initially, the examiners will evaluate a few articles separately (pilot study), using the examination questionnaire, to test their effectiveness. The two reviewers (BP and SK) will then independently evaluate the retrieved articles using a screening questionnaire. In case of uncertainty regarding the incorporation of the 
article (after checking the title and the abstract), the full version of the text will be retrieved and checked. A third examiner (PA) will intervene only in cases of doubt. Recovered publications will be imported into the Endnote software package.

\section{Data extraction process}

An appropriate form will be configured to collect the data of the selected articles. Specific study information will be collected by the first examiner (BP). The second examiner (SK) will check the accuracy of the procedure. Some studies may have incomplete data, so we will encourage communication with their authors. Given the rigorous curriculum, the authors will have 2 weeks to answer our questions. Otherwise, the study will be described as "vague". The elements that we will gather from the selected studies, include the construct, population, type of measurement instrument, measurement properties of interest and the statistics (the model used, the result and its $95 \%$ confidence interval).

\section{Risk of bias assessment}

Assessing the quality of primary studies is an important part of a systematic review to assess the risk of bias. Understanding the quality of the studies and the measuring instrument used is a difficult task. To this end, the COSMIN checklist was developed, a standard tool for assessing the risk of bias in studies. ${ }^{31}$ The COSMIN checklist was originally developed for self-reported evaluation tools, but now its revised format has been extended to other tools, too. ${ }^{32}$ In this review, two reviewers (BP and SK) will independently evaluate the psychometric characteristics (reliability, validity and measurement error, criterion validity, hypotheses testing for construct validity and responsiveness) (Table 3) checklist in a four-point scoring system (very good, adequate, doubtful and inadequate) of each study, based on the standards from COSMIN.

According to the instructions we will use the lower score counts methods to come to an overall rating per study. The third examiner (PA) will intervene again in case of doubt.

\section{Synthesis of results}

The next step is to summarize the data. If at least three studies with relevant data are available for each QST component in the shoulder and with sufficient homogeneity, the attempt for meta-analysis using a random effects model will be encouraged. Otherwise, the quality of the evidence for each measurement tool and method will be separately evaluated. The criteria for each of the estimated measurement properties for each instrument and the measurement method will be marked as sufficient (+), unspecified (?) or insufficient (-) (Table 4). Finally, the modified grading of recommendations assessment, development, and evaluation (GRADE) approach will be used to assess the overall quality of the evidence as high, medium, low or very low, based on the risk of bias, inconsistency, imprecision, and indirectness. ${ }^{32}$

Table 2: Search strategy for PubMed.

\begin{tabular}{|c|c|}
\hline \multicolumn{2}{|c|}{ Search strategy } \\
\hline \#1 & 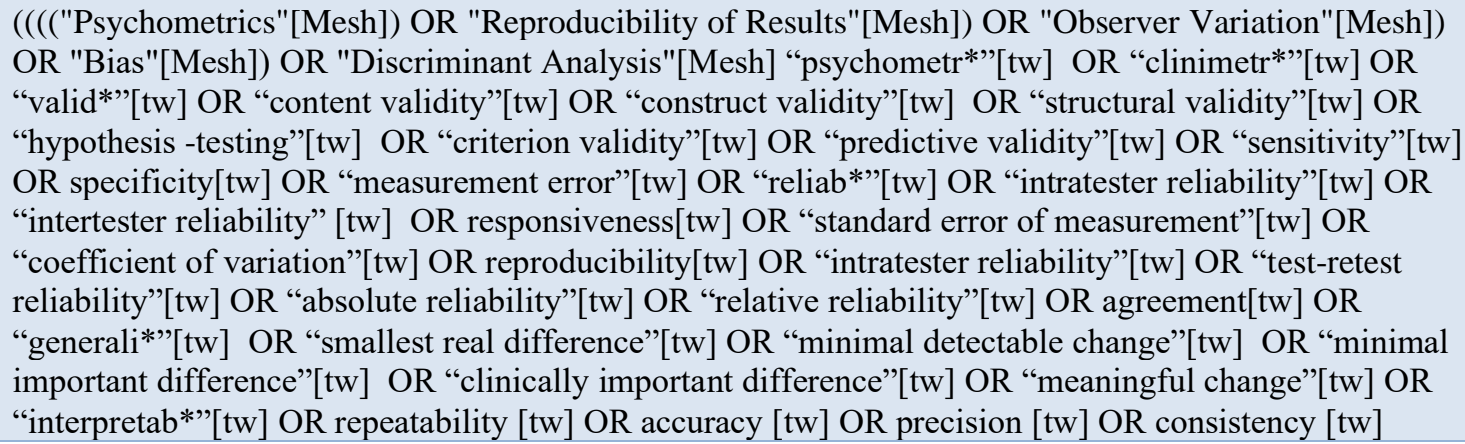 \\
\hline \#2 & 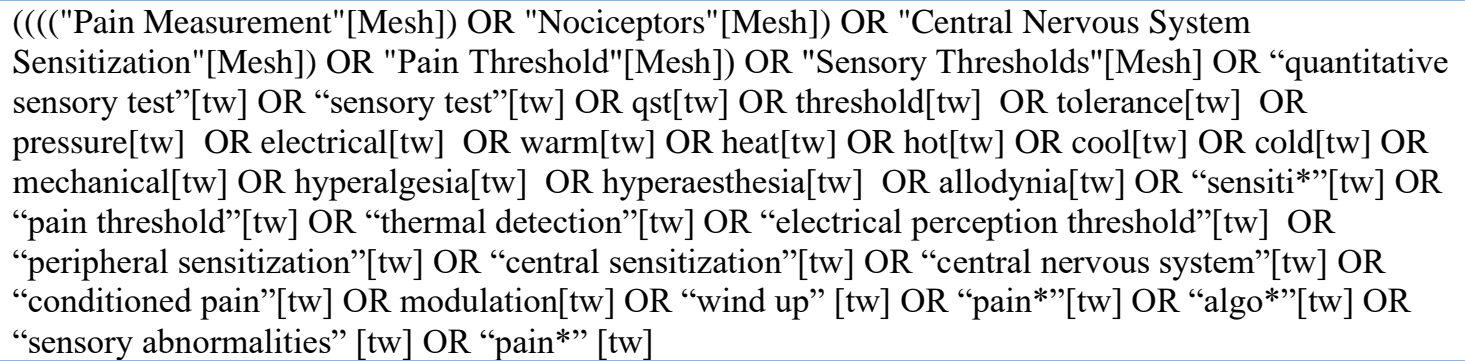 \\
\hline \#3 & ("Shoulder"[Mesh] OR "Shoulder Pain"[Mesh]) OR “shoulder*” \\
\hline \#4 & \#1 AND \#2 AND \#3 \\
\hline
\end{tabular}


Table 3: Definitions of measurement property adapted from COSMIN recommendation (except where indicated) (*definitions adapted from other sources).

\begin{tabular}{|c|c|c|c|}
\hline \multicolumn{3}{|l|}{ Term } & \multirow[b]{2}{*}{ Definition } \\
\hline Domain & $\begin{array}{l}\text { Measurement } \\
\text { property }\end{array}$ & $\begin{array}{l}\text { Subsets of } \\
\text { measurement } \\
\text { property }\end{array}$ & \\
\hline $\begin{array}{l}\text { Reliability } \\
\text { (extended } \\
\text { definition) }\end{array}$ & & & $\begin{array}{l}\text { The degree to which the results for participants, are the similar for } \\
\text { repeated measurements of QST (in the same group under several } \\
\text { conditions) and free from measurement error. }\end{array}$ \\
\hline & Test-retest & & $\begin{array}{l}\text { The extent of agreement in repeated measurements of QST over } \\
\text { time. }\end{array}$ \\
\hline & Intra-rater & & $\begin{array}{l}\text { The extent of agreement in repeated measurements of QST on the } \\
\text { same participant by the same rater. }\end{array}$ \\
\hline & Inter-rater & & $\begin{array}{l}\text { The extent of agreement between raters investigating sensory } \\
\text { abnormalities scores with QST on the same individual. }\end{array}$ \\
\hline & $\begin{array}{l}\text { Measurement } \\
\text { error }\end{array}$ & & $\begin{array}{l}\text { The systematic and random error of a participant's score that is not } \\
\text { attributed to true changes in sensory to be measured. }\end{array}$ \\
\hline Validity & & & $\begin{array}{l}\text { The degree to which a component of QST measures the sensory } \\
\text { abnormalities i.e., the construct which has designed to measure. }\end{array}$ \\
\hline & $\begin{array}{l}\text { Construct } \\
\text { validity }\end{array}$ & & $\begin{array}{l}\text { The extent to which the scores of a specific sensory test of QST are } \\
\text { consistent with hypotheses regarding the scores of other } \\
\text { measurement methods or differences between known groups, given } \\
\text { that QST validly measures the construct it is purported to measure. }\end{array}$ \\
\hline & & $\begin{array}{l}\text { Discriminative/ } \\
\text { known groups } \\
\text { validity }\end{array}$ & $\begin{array}{l}\text { The degree to which the scores of a specific test of QST can } \\
\text { discriminate between groups known to differ in sensibility (i.e. } \\
\text { individuals with SIS versus healthy individuals). }\end{array}$ \\
\hline & & $\begin{array}{l}\text { Convergent and } \\
\text { discriminant } \\
\text { (divergent) } \\
\text { validity*33 }\end{array}$ & $\begin{array}{l}\text { The specific test of QST show conceptual convergence or } \\
\text { divergence between them or with other outcome measures } \\
\text { attributed to different constructs such as pain intensity, disability, } \\
\text { depression etc. }\end{array}$ \\
\hline & $\begin{array}{l}\text { Criterion } \\
\text { validity }\end{array}$ & $\begin{array}{l}\text { Predictive } \\
\text { validity }\end{array}$ & $\begin{array}{l}\text { The degree of correlation to a reference standard (i.e. a PRO) } \\
\text { measuring sensibility with a specific test of QST (i.e. PPT). }\end{array}$ \\
\hline $\begin{array}{l}\text { Responsive } \\
\text { ness }\end{array}$ & & & $\begin{array}{l}\text { The ability of a special test of QST to detect change in sensibility } \\
\text { over time }\end{array}$ \\
\hline $\begin{array}{l}\text { Sensitivity* } \\
34\end{array}$ & & & $\begin{array}{l}\text { The proportion of individuals with shoulder pain (affected) } \\
\text { identified with sensory abnormalities }\end{array}$ \\
\hline Specificity* & & & $\begin{array}{l}\text { The proportion of healthy individuals (unaffected) identified } \\
\text { without sensory abnormalities }\end{array}$ \\
\hline
\end{tabular}

Table 4: Criteria for evaluating the quality of the psychometric properties according to the COSMIN checklist.

\begin{tabular}{|l|l|}
\hline Measurement property/rating & Criteria \\
\hline Reliability & ICC or weighted kappa $\geq 0.70$ \\
\hline Sufficient (+) & ICC or weighted kappa not reported \\
\hline Indeterminate (?) & ICC or weighted kappa $<0.70$ \\
\hline Insufficient (-) & SDC or LoA $<$ MIC \\
\hline Measurement error & MIC not defined \\
\hline Sufficient (+) & SDC or LoA $>$ MIC \\
\hline Indeterminate (?) & \\
\hline Insufficient (-) & Correlation with gold/reference standard $\geq 0.70$ or AUC $\geq 0.70$ \\
\hline Criterion validity & Not all information for “+” reported \\
\hline Sufficient (+) & Correlation with gold/reference standard $<0.70$ or AUC $<0.70$ \\
\hline Indeterminate (?) & \\
\hline Insufficient (-) & Agreement with the hypothesis or AUC $\geq 0.70$ \\
\hline Responsiveness & \\
\hline Sufficient (+) & \\
\hline
\end{tabular}




\begin{tabular}{|l|l|}
\hline Measurement property/rating & Criteria \\
\hline Indeterminate (?) & Hypothesis is NOT defined (by the review committee) \\
\hline Insufficient $(-)$ & Not agreement with the hypothesis or AUC $<0.70$ \\
\hline Sensitivity and specificity & \\
\hline Sufficient (+) & Sensitivity and specificity calculated \\
\hline Indeterminate (?) & Not applicable \\
\hline Insufficient (-) & Sensitivity and specificity NOT calculated \\
\hline
\end{tabular}

$\mathrm{AUC}=$ Area under the care, $\mathrm{SDC}=$ smallest detectable change, $\mathrm{LoA}=$ limits of agreement, $\mathrm{MIC}=$ minimal important change

\section{DISCUSSION}

To our knowledge, this is the first systematic review investigating the PMPs of QST specifically targeting the shoulder. Only one study assessed the level of evidence for PMP testing procedures in people with pain in peripheral joints, including the shoulder. However, the literature search in this study was conducted in seven databases from inception to March 2016. In this search, the gap from the previous review will be filled. The limitations of this protocol include the acceptance of original research articles published in English and Greek. Studies that have not been published in peer-reviewed journals (eg results from conference presentations, chapters and book reviews, doctoral and other dissertations) will be rejected. This protocol was written in accordance with the recommendations provided by Preferred Reporting Items for Systematic Reviews and Meta-Analysis (PRISMA) and Consensus-Based Standards for the Selection of Health Measurement Instruments (COSMIN). The results will allow clinicians to make an informed evidence-based assessment and will inform further research into this topic.

\section{CONCLUSION}

To date, although the use of QST is common in assessment of patients with chronic shoulder pain, the level of evidence for psychometric properties has not been documented. Evaluation of the level of evidence for the psychometric properties of QST in the shoulder is an essential step for evidence-based assessment in clinical practice.

Funding: The study was funded by Research, Innovation and Excellence Structure (DEKA) of the University of Thessaly

Conflict of interest: None declared

Ethical approval: The study was approved by the Institutional Ethics Committee

\section{REFERENCES}

1. Djade CD, Porgo TV, Zomahoun HTV, PerraultSullivan G, Dionne CE. Incidence of shoulder pain in 40 years old and over and associated factors: A systematic review. Eur J Pain. 2020;24(1):39-50.

2. Menendez ME, Baker DK, Oladeji LO, Fryberger CT, McGwin G, Ponce BA. Psychological Distress Is Associated with Greater Perceived Disability and Pain in Patients Presenting to a Shoulder Clinic. J Bone Joint Surg Am. 2015;97(24):1999-2003.
3. Imagama S, Ando K, Kobayashi K. Shoulder pain has most impact on poor quality of life among various types of musculoskeletal pain in middle-aged and elderly people: Yakumo study. Modern Rheumatology. 2020;30:568-72.

4. Liu R, Onks C, Petscavage-Thomas J, Walker E, Berg A, Silvis M, Dhawan A. Prevalence of shoulder labral abnormalities on MRI in a non-athletic asymptomatic young adult population. Skeletal Radiol. 2021;50(5):921-5.

5. Schwartzberg R, Reuss BL, Burkhart BG. High Prevalence of Superior Labral Tears Diagnosed by MRI in Middle-Aged Patients With Asymptomatic Shoulders. Orthopaedic journal of sports medicine. 2016;4:2325967115623212.

6. Chimenti RL, Frey-Law LA, Sluka KA. A Mechanism-Based Approach to Physical Therapist Management of Pain. Physical therapy. 2018;98:30214.

7. Harte SE, Harris RE, Clauw DJ. The neurobiology of central sensitization. Journal of Behavioral Research. 2018;23:12137.

8. Smart KM, Blake C, Staines A, Doody C. The Discriminative validity of "nociceptive," "peripheral neuropathic," and "central sensitization" as mechanisms-based classifications of musculoskeletal pain. Clin J Pain. 2011;27(8):655-63.

9. Cruz-Almeida Y, Fillingim RB. Can quantitative sensory testing move us closer to mechanism-based pain management? Pain medicine (Malden, Mass). 2014;15:61-72.

10. Rolke R, Magerl W, Campbell KA. Quantitative sensory testing: a comprehensive protocol for clinical trials. European journal of pain (London, England). 2006;10:77-88.

11. Arendt-Nielsen L, Morlion B, Perrot S. Assessment and manifestation of central sensitisation across different chronic pain conditions. 2018;22:216-41.

12. Uddin Z, MacDermid JC. Quantitative Sensory Testing in Chronic Musculoskeletal Pain. Pain medicine (Malden, Mass). 2016;17:1694-703.

13. Hall TB, Schafer A, Tampin B, Moloney N. Quantitative sensory testing: implications for clinical practice. In: Jull G MA, Falla D, Lewis J, McCarthy C, Sterling M, (ed) Grieve's modern musculoskeletal physiotherapy. 4th ed. United Kingdom: Elsevier. 2015;194-201.

14. Hidalgo-Lozano A, Fernandez-de-las-Penas C, Alonso-Blanco C, et al. Muscle trigger points and pressure pain hyperalgesia in the shoulder muscles in patients with unilateral shoulder impingement: a 
blinded, controlled study. Experimental brain research. 2010;202:915-25.

15. Paul TM, Soo Hoo J, Chae J, Wilson RD. Central hypersensitivity in patients with subacromial impingement syndrome. Arch Phys Med Rehabil. 2012;93(12):2206-9.

16. Alagappan T, Senthilkumar S, Dhanani D. Recognition of central nervous system sensitization and its risk factors in patients with unilateral musculoskeletal shoulder pain. 2019;13:102-8.

17. Arnstad ED, Iversen JM, Uglem M, Glerup M, Romundstad PR, Sand T, Rygg M. Pain sensitivity in young adults with juvenile idiopathic arthritis: a quantitative sensory testing study. Arthritis Res Ther. 2020;22(1):262.

18. Smith SM, Dworkin RH, Turk DC, Baron R, Polydefkis M, Tracey I, et al. The Potential Role of Sensory Testing, Skin Biopsy, and Functional Brain Imaging as Biomarkers in Chronic Pain Clinical Trials: IMMPACT Considerations. J Pain. 2017;18(7):757-77.

19. van Helmond N, Aarts HM, Timmerman H. Is Preoperative Quantitative Sensory Testing Related to Persistent Postsurgical Pain? A Systematic Literature Review. Anesthesia \& Analgesia. 2020;131:1146-55.

20. Giannoni-Luza S, Pacheco-Barrios K, CardenasRojas A. Noninvasive motor cortex stimulation effects on quantitative sensory testing in healthy and chronic pain subjects: a systematic review and metaanalysis. 2020;161:1955-75.

21. Tampin B, Slater H, Jacques A, Lind CRP. Association of quantitative sensory testing parameters with clinical outcome in patients with lumbar radiculopathy undergoing microdiscectomy. Eur J Pain. 2020;24(7):1377-92.

22. Clark J, Nijs J, Yeowell G, Goodwin PC. What Are the Predictors of Altered Central Pain Modulation in Chronic Musculoskeletal Pain Populations? A Systematic Review. Pain Physician. 2017;20(6):487500 .

23. Georgopoulos V, Akin-Akinyosoye K, Zhang W. Quantitative sensory testing and predicting outcomes for musculoskeletal pain, disability, and negative affect: a systematic review and meta-analysis. Pain. 2019;160:1920-32.

24. Alqarni AM, Manlapaz D, Baxter D. Test Procedures to Assess Somatosensory Abnormalities in Individuals with Peripheral Joint Pain: A Systematic Review of Psychometric Properties. Pain practice : the official journal of World Institute of Pain. 2018;18:895-924.

25. Kennedy DL, Kemp HI, Ridout D. Reliability of conditioned pain modulation: a systematic review. Pain. 2016;157:2410-9.
26. Geber C, Klein T, Azad S, Birklein F, Gierthmühlen $\mathrm{J}$, Huge $\mathrm{V}$, et al. Test-retest and interobserver reliability of quantitative sensory testing according to the protocol of the German Research Network on Neuropathic Pain (DFNS): a multi-centre study. Pain. 2011;152(3):548-56.

27. Moloney NA, Hall TM, Doody CM. Reliability of thermal quantitative sensory testing: a systematic review. Journal of rehabilitation research and development. 2012;49:191-207.

28. Marcuzzi A, Dean CM, Wrigley PJ, Chakiath RJ, Hush JM. Prognostic value of quantitative sensory testing in low back pain: a systematic review of the literature. J Pain Res. 2016;9:599-607.

29. Moher D, Shamseer L, Clarke M, Ghersi D, Liberati A, Petticrew M, Shekelle P, Stewart LA; PRISMA-P Group. Preferred reporting items for systematic review and meta-analysis protocols (PRISMA-P) 2015 statement. Syst Rev. 2015;4(1):1.

30. Shamseer L, Moher D, Clarke M, Ghersi D, Liberati A, Petticrew M, Shekelle P, Stewart LA; PRISMA-P Group. Preferred reporting items for systematic review and meta-analysis protocols (PRISMA-P) 2015: elaboration and explanation. BMJ. 2015;350:7647.

31. Mokkink LB, de Vet HCW, Prinsen CAC, Patrick DL, Alonso J, Bouter LM, Terwee CB. COSMIN Risk of Bias checklist for systematic reviews of Patient-Reported Outcome Measures. Qual Life Res. 2018;27(5):1171-9.

32. Prinsen CAC, Mokkink LB, Bouter LM, Alonso J, Patrick DL, de Vet HCW, Terwee CB. COSMIN guideline for systematic reviews of patient-reported outcome measures. Qual Life Res. 2018;27(5):114757.

33. Souza ACD, Alexandre NMC, Guirardello EdB. Propriedades psicométricas na avaliação de instrumentos: avaliação da confiabilidade e da validade. J Epidemiologia e Serviços de Saúde. 2017;26:649-59.

34. Parikh R, Mathai A, Parikh S, Sekhar GC, Thomas R. Understanding and using sensitivity, specificity and predictive values. Indian journal of ophthalmology. 2008;56:45-50.

Cite this article as: Bilika $\mathrm{P}$, Savvoulidou K, Paliouras A, Dimitriadis Z, Billis E, Strimpakos N, et al. Psychometric properties of quantitative sensory testing focusing on healthy and patients with shoulder pain: a systematic review protocol. Int J Clin Trials 2021;8(3):253-9. 